Check for updates

Cite this: RSC Adv., 2018, 8, 2622

Received 3rd October 2017

Accepted 19th December 2017

DOI: 10.1039/c7ra10904k

rsc.li/rsc-advances

\section{Yeast fermentation inspired Ca-alginate hydrogel membrane: lower transparency, hierarchical pore structure and higher hydrophobicity $\dagger$}

\author{
Lijuan Xing, ${ }^{a}$ Zhigang Li, ${ }^{a}$ Qingsong Zhang, (D) *a Yixuan Zhang, ${ }^{a}$ Pengfei Liu ${ }^{b}$ \\ and Kailin Zhang ${ }^{\mathrm{C}}$
}

With a fantastic combination of yeast fermentation and hydrogel membrane formation, a series of microorganism inspired porous hydrogel membranes (MIHM) with various mass ratios of yeast/ sodium alginate (SA) were developed. The yeast cells were firstly activated by addition of glucose for generating byproduct carbon dioxide $\left(\mathrm{CO}_{2}\right)$, inducing the formation of porous structures. The ionic cross-linking between calcium chloride and SA was subsequently performed based on an egg-box model. It is of interest to note that the obtained MIHM hydrogel membranes exhibited decreased transparency and hierarchical porous structure with pore sizes varying from $2 \mathrm{~nm}$ to $1 \mathrm{~mm}$, giving rise to significantly increased contact angle (CA) values from $63.65^{\circ}$ to $107.83^{\circ}$ and obviously decreased equilibrium swelling ratios from 79.72 to 18.05. Especially, the adsorption kinetics of crystal violet $(\mathrm{CV})$ show that the MIHM hydrogel membrane exhibited an encouraging uptake capacity of $18.40 \mathrm{mg} \mathrm{g}^{-1}$ at $10 \mathrm{mg} \mathrm{g}^{-1}$ and the adsorption kinetics of CV could be well defined by a pseudo-firstorder kinetic equation. Owing to great advantages in facile fabrication, high efficiency, low cost and desirable biocompatibility, the MIHM hydrogel membrane can be large-scale fabricated and used for industry and agriculture.

\section{Introduction}

The pore characteristics of hydrogels, like pore structure, pore size and porosity, play a key role in controlling water absorbance capacity, swelling rates, mechanical strength, separation efficiency, sensitivity degree, and so forth. Many methods have been used to form porous hydrogels, such as the pore forming agent method, emulsion templating method, ice-templated assembly and phase separation method..$^{1-4}$ However, some inherent problems limited their extensive application. For example, Wang et al. prepared nano-porous hydrogels using micelle/emulsion as the pore-forming template, but the operation process is too complicated. ${ }^{5}$ Gyarmati et al. fabricated chemically cross-linked poly(aspartic acid) gels by a solid-liquid phase separation to achieve a pore structure, but the adopted solvent dimethyl sulfoxide is poisonous. ${ }^{6}$ On the other hand, in

${ }^{a}$ State Key Laboratory of Separation Membranes and Membrane Processes, State Key Laboratory of Hollow Fiber Membrane Materials and Processes, School of Materials Science and Engineering, Tianjin Polytechnic University, Tianjin 300387, China. E-mail: zqs8011@163.com

${ }^{b}$ School of Computer Science \& Software Engineering, Tianjin Polytechnic University, Tianjin 300387, China

${ }^{c}$ College of Chemical and Environmental Engineering, Shandong University of Science and Technology, Tsingtao 266590, China

$\dagger$ Electronic supplementary information (ESI) available. See DOI: 10.1039/c7ra10904k comparison to three dimensional (3D) gels, 2D hydrogel membranes show rapid swelling and adsorption behavior due to high specific surface area. Consequently, it is particularly necessary to develop a simple, effective and environmentalfriendly method to prepare porous hydrogels membrane.

In consideration of environmental friendliness to the earth, the exploration of polymer membrane materials from renewable resources has drawn significant attention. As a natural anionic polyelectrolyte, sodium alginate (SA) has several remarkable features like low cost, biodegradability, biocompatibility, simplicity to use and capability to undergo chainchain association and to form three dimensional gels in the presence of divalent cations, making it a desirable choice of material to be applied in adsorption, drug delivery, cell culture, scaffold and separation..$^{7-10}$ Owing to good membrane forming properties, SA has been widely used in recent years. To protect against wet-out, $\mathrm{Xu}$ et al. fabricated the polytetrafluoroethylene (PTFE) membranes coated with SA hydrogel, observing that the coated membrane retained its integrity after contacting with a $1.2 \mathrm{wt} \%$ oil-water mixture for $72 \mathrm{~h} .{ }^{11}$ Kulkarni et al. prepared interpenetrating polymer networks (IPN) hydrogel membranes of SA and polyvinyl alcohol (PVA) by solvent casting method, proving good safety for transdermal delivery of an antihypertensive drug. ${ }^{12}$ Zhang et al. synthesized an anti-fouling, free-standing membrane through the polymerization of acrylamide (AM) in the presence of SA. ${ }^{13}$ The results showed that the 
prepared calcium alginate/polyacrylamide (CA/PAM) membrane exhibited $89.06 \%$ and $85.84 \%$ water flux recovery for yeast suspension and bull serum albumin (BSA) solution, respectively. However, in most cases, these materials do not exhibit satisfying adsorption performance. Therefore, it appears especially important to develop a novel membrane with high adsorption capacity.

As is universally known, the yeast species can convert carbohydrates to carbon dioxide $\left(\mathrm{CO}_{2}\right)$ and alcohols. The carbon dioxide from yeast fermentation has been used in baking and the alcohol production for tens of thousands of years. In our previous work, a method to prepare porous bulk PAM hydrogel membrane inspired by yeast fermentation has been established. ${ }^{14,15}$ That is, taking advantage of fermentation process in microbiology, the unicellular fungus yeast is employed to generate pores in hydrogel. One the one hand, this kind of 3D hydrogel shows lower swelling ratio and adsorption capacity. On the other hand, only a few studies upon compressive and tensile behavior of yeast cell-loaded alginate hydrogel constructions were published. ${ }^{16,17}$ With consideration of multifunctional group of SA, rapid pore formation of yeast fermentation and increased surface area of 2D membrane, porous Caalginate hydrogel membrane with porous structure fabricated by yeast fermentation was proposed.

In present work, a novel porous yeast/glucose $/ \mathrm{SA} / \mathrm{CaCl}_{2}$ hydrogel membrane (Microorganism Inspired Hydrogel Membrane, abbreviated as MIHM) was prepared using yeast/ glucose as porogen. Compared to other methods, preparation of porous hydrogels by adding yeast shows apparent advantages, such as simplified operation process, relatively inexpensive agents, rapid pore formation, large specific surface area and high adsorption capacity. The optical performances of MIHM hydrogel membranes before and after purification were investigated by UV/vis. The pore shape, size and porosity of prepared MIHM hydrogel membranes were observed by environmental scanning electron microscopy (ESEM), mercury intrusion porosimetry (MIP) and Brunauer, Emmett \& Teller (BET). The surface hydrophilic/hydrophobic property and swelling behavior of the hydrogel membranes were analyzed by contact angle measurement and gravimetric method. The adsorption behavior of MIHM hydrogel membranes for removing crystal violet (CV) was also investigated. The pore size could be controlled by adjusting yeast and glucose concentration. The produced porous SA/ $\mathrm{CaCl}_{2}$ hydrogel membranes have great potential applications in the field of industry and agriculture like chemical adsorption.

\section{Experimental}

\subsection{Materials}

Both sodium alginate $(\mathrm{SA})$ and calcium chloride $\left(\mathrm{CaCl}_{2}\right)$ were purchased from Tianjin Kemiou Chemical Reagent Co., Ltd. Instant dry yeast was obtained from Angel Yeast Co., Ltd. Glucose was purchased from Tianjin Fengchuan Chemical Reagent Co., Ltd. As a water-soluble monovalent cationic dye, crystal violet (CV, Tianjin Chemical Reagent Co., Ltd, analytical reagent grade) was used as received. Water was deionized by Molecular apparatus (Chongqing Molecular Water System Co. Ltd. China).

\subsection{Preparation of porous yeast/glucose/ $\mathrm{SA} / \mathrm{CaCl}_{2}$ hydrogel membrane}

$0.1 \mathrm{~g}$ yellow SA powder was firstly dissolved in $10 \mathrm{~mL}$ distilled water until the formation of transparent solution. Rehydration solution was obtained by dissolving glucose and instant dry yeast into $10 \mathrm{~mL}$ distilled water for 5 minutes under constant stirring. The mass ratio of glucose/yeast was controlled at $1 / 3$. The rehydration solution was subsequently added into SA aqueous solution slowly at a constant stirring speed to form homogenous solution. Thereafter, the generated solution was poured into glass mold with $1.0 \mathrm{~mm}$ thickness. Finally, the glass mold was put into $100 \mathrm{mM} \mathrm{CaCl}_{2}$ solution for ionic crosslinking at $35^{\circ} \mathrm{C}$. The whole reaction lasted nearly $4 \mathrm{~h}$.

After ionic crosslinking, the resultant hydrogel membranes were taken out from glass molds before cutting into $1 \mathrm{~cm}$ discs in diameter with punch, and immersed in excess water for 7 days to remove $\mathrm{Ca}^{2+}$, other small molecules and dead or live yeast cells. The formed hydrogel membranes were simply named as MIHMX. MIHM means Microorganism Inspired Hydrogel Membrane, and the mass ratio of yeast/SA is expressed as X. For example, the hydrogel membrane synthesized by $0.5 \mathrm{~g}$ yeast and $0.1 \mathrm{~g}$ SA is expressed as MIHM5.

\subsection{Surface and cross-section shape observation of hydrogel membrane}

To keep original pore structure, the MIHM hydrogel membrane specimens were freeze-dried at $-50{ }^{\circ} \mathrm{C}$ for $12 \mathrm{~h}$ on a FD-1A-50 freeze-drier (Beijing Bilon Experimental Equipment Co. Ltd.) so that water in the hydrogel membrane could be completely removed. The surface morphologies of specimens were observed on an environmental scanning electron microscope (ESEM, Quanta 200, FEI) after coated with gold under vacuum. It needs to be noted that the samples at wet state can't be observed on account of operation requirement.

The swollen MIHM hydrogel discs in deionized water were freeze-dried at $-50{ }^{\circ} \mathrm{C}$ for $12 \mathrm{~h}$ on a $\mathrm{FD}-1 \mathrm{~A}-50$ freeze-drier instrument to completely remove any water for the FESEM analysis. Cross-section samples were obtained by freezefractured in liquid nitrogen and then sputtered with gold. Hydrogel cross-section were observed using a field emission scanning electron microscopy (S-4800, HITACHI, Japan) after sputter-coating with gold under vacuum.

\subsection{Hydrophilicity and hydrophobicity of hydrogel membrane}

The MIHM hydrogel membrane samples were firstly placed on a clean glass until forming of dried membrane. Then the static CA values of MIHM hydrogel membranes were measured on a DSA100 contact angle meter (Kruss Instrument Co., Germany). To reduce the error, each group was repeated for three times and the mean value was calculated. 


\subsection{Hydrophilicity and hydrophobicity of hydrogel} membrane

The optical transmittance values of MIHM hydrogel membranes before and after purification were investigated by ultraviolet visible spectroscopy (UV/vis, TU-1901, Beijing Purkinje General Instrument Co., Ltd) under the maximum absorbance value. Here, purification was made according to the method described.

\subsection{Pore structure and basic properties analysis of hydrogel membrane}

On the one hand, the porosity ratio, pore volume and pore size distribution of the MIHM hydrogel membranes were determined by mercury intrusion porosimetry (MIP, AutoPore IV 9500, Micromeritics Instrument Co. Ltd., USA). The determination of porosity features was based on the Washburn equation.

On the other hand, the pore volume, pore diameter and specific surface areas were determined by nitrogen adsorption/ desorption isotherms with standard Brunauer-Emmett-Teller (BET) (Autosorb-1, Quantachrome Instrument Corporation, USA). Prior to the adsorption experiments, the MIHM hydrogel membranes were heated $\left(25^{\circ} \mathrm{C}\right)$ under vacuum for $12 \mathrm{~h}$. Data were analyzed by a computer controlled sorption analyzer (Autosorb-1 Software) operated in the continuous mode. Data reduction software programs included BET surface area analysis and $t$-plot analysis (with reference to a nonporous silica and micropore).

\subsection{Swelling behavior of hydrogel membrane}

A gravimetric procedure was adopted to monitor the maximum swelling ratios (MSR) of MIHM hydrogel membranes. In brief, swollen MIHM hydrogel samples with disc shape were taken out and wiped with filter paper to remove excess water before weighing. The measurements were carried out until a constant weight was reached for each sample. The MSR values were calculated by eqn (1),

$$
\operatorname{MSR}=\frac{M_{\mathrm{wet}}-M_{\mathrm{dry}}}{M_{\mathrm{dry}}}
$$

where $M_{\mathrm{dry}}$ is the weight of dried MIHM hydrogel membrane samples, and $M_{\text {wet }}$ is the weight of water-swollen MIHM hydrogel membrane samples after removing excess water from the surface at $25^{\circ} \mathrm{C}$, respectively.

\subsection{Adsorption kinetics of MIHM hydrogel membranes on CV}

To investigate the effects of CV contact time on the adsorption capacity, all MIHM samples were immersed into $30 \mathrm{~mL}$ of $\mathrm{CV}$ aqueous solution with the concentration $10 \mathrm{mg} \mathrm{L}{ }^{-1}$ at $25{ }^{\circ} \mathrm{C}$. During the adsorption, CV solution was withdrawn from the adsorption system at the indicated time for analyzing CV concentrations by a UV/vis spectrophotometer (TU-1901) at $\lambda_{\max }$ $=584 \mathrm{~nm}$. For each sample, the withdrawn solution was put back into the test system. The amounts of CV adsorbed by the
MIHM hydrogel membrane at time $t, q_{t}$, were determined according to eqn (2),

$$
q_{t}=\frac{\left(C_{0}-C_{t}\right) V}{m}
$$

where $V$ is the solution volume $(\mathrm{mL}), m$ is the weight of freezedried MIHM hydrogel membrane samples (g), $C_{0}$ and $C_{t}$ is the $\mathrm{CV}$ concentration at initial and indicated time $\left(\mathrm{mg} \mathrm{mL} \mathrm{mL}^{-1}\right)$, respectively.

\section{Results and discussion}

\subsection{The appearance and optical performance of MIHM hydrogel membranes}

The appearances of MIHM hydrogel membranes are shown in Fig. $1 \mathrm{a}-\mathrm{d}$. It is seen that pure $\mathrm{SA} / \mathrm{CaCl}_{2}$ hydrogel membrane shown in Fig. 1a presents good transparency and any pore structure can not be found on the surface. However, after freezedried, the pure $\mathrm{SA} / \mathrm{CaCl}_{2}$ hydrogel membrane turns white due to the evaporation of water molecules at $-50{ }^{\circ} \mathrm{C}$, as shown in Fig. 1b. It is of interest to note that with the introduction of yeast/glucose, the surfaces of MIHM5 and MIHM15 hydrogel membrane samples exhibit obvious porous structure and significantly low transparency, which can be seen by the naked eye. Actually, although yeast cells have been fermented, dead cells still remain at the matrix of MIHM hydrogel membranes, which have been confirmed as shown in Fig. S1. $\dagger$ In terms of pore size, sample MIHM15 shows bigger pore size than sample MIHM5. In other words, with increasing the mass ratios of yeast/SA from 5 to 15 , the pore size of MIHM hydrogel membrane increases. The reason lies in that high yeast/glucose content could produce a large amount of $\mathrm{CO}_{2}$ during fermentation.

To better understand the influence of yeast on optical performance, UV/vis was used to examine the transmittance of MIHM hydrogel membranes before and after purification, as displayed in Fig. 1e. According to full scanning results of MIHM hydrogel membranes between $200 \mathrm{~nm}$ and $900 \mathrm{~nm}$, the maximum absorption peak occurs at $679 \mathrm{~nm}$. It is seen that, before purification, the absorbance values of MIHM hydrogel membranes rapidly increase from 0.23 to 2.63 with mass ratios of yeast/SA increased from 0 to 15 . Unexpectedly, after immersing in deionized water for $48 \mathrm{~h}$, the absorbance values of MIHM hydrogel membranes increase from 0.44 to 2.42 . The increasing absorbance value of MIHM0 after purification can be attributed to the increased inflective index caused by bigger pore size after absorbing water. Accordingly, two conclusions can be drawn from the curves. On the one hand, the introduction of yeast cells decreases the transparency of MIHM hydrogel membranes, which is consistent with Fig. 1a-d. On the other hand, some dead yeast cells and impurity could escape from the three-dimensional $\mathrm{SA} / \mathrm{CaCl}_{2}$ hydrogel network after immersing in deionized water. Similar result has been observed by Gao et al. that the introduction of surfactant polyoxyethylene (20) cetyl ether (Brij-58) could rapidly decrease the transmittance of reaction mixture solution. ${ }^{\mathbf{1 8}}$ In fact, in spite of the reduced transparency after purification, MIHM hydrogel membranes 

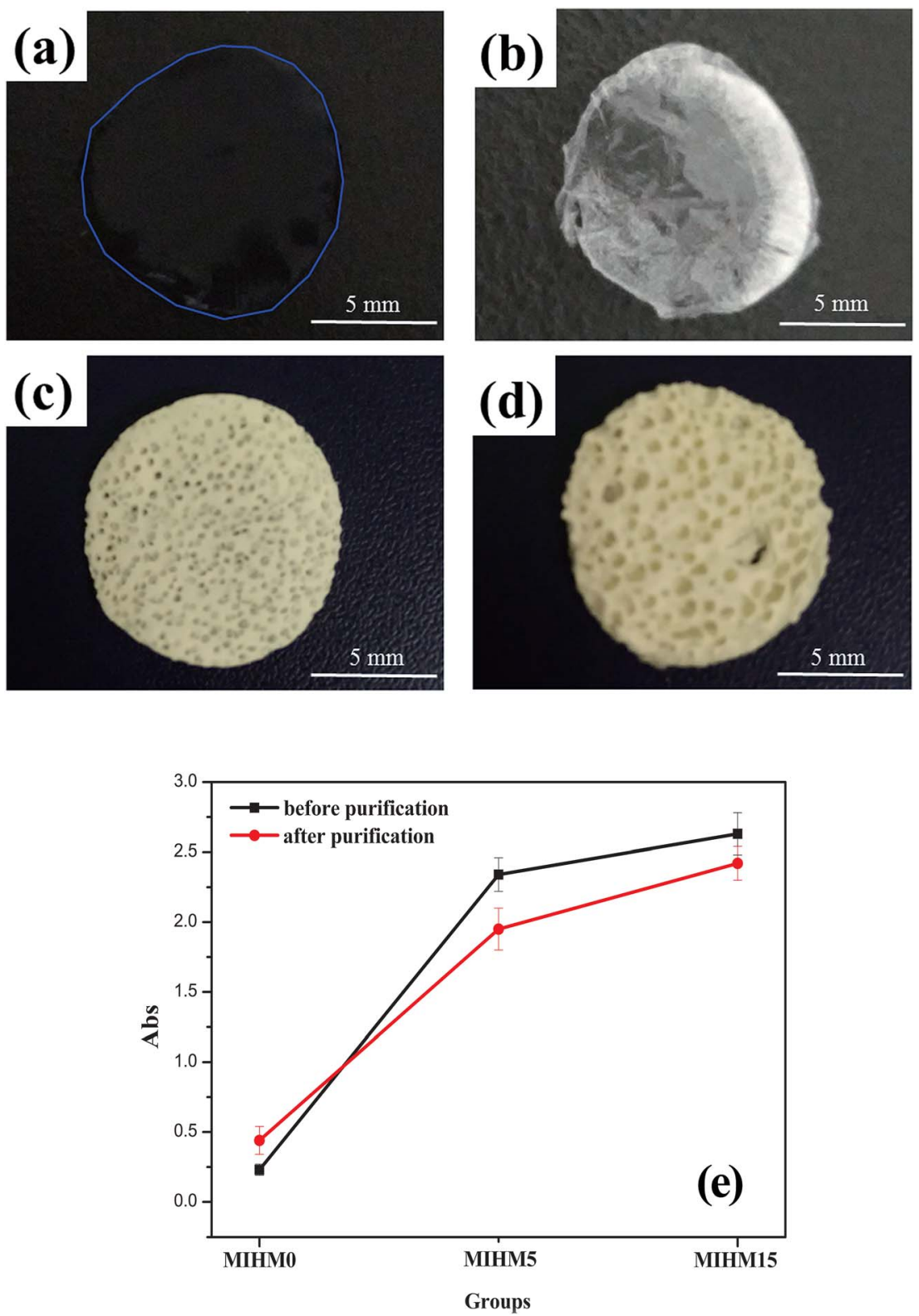

Fig. 1 Appearance of MIHM hydrogel membranes: (a) water-swollen MIHMO hydrogel membrane, (b) freeze-dried MIHMO hydrogel membrane, (c) freeze-dried MIHM5 hydrogel membrane and (d) freeze-dried MIHM15 hydrogel membrane, and (e) curves of absorbance values of MIHM before and after purification.

after fermentation still keep low light penetration on account of great difficulty in removing plenty of dead yeast cells from the matrix of hydrogel membranes.

\subsection{Fabrication and formation mechanism of porous MIHM hydrogel membrane}

Schematic representation for the preparation of porous MIHM is depicted in Fig. 2. To fabricate porous hydrogel membrane, the entire experiment process can be divided into two steps. In the first step, the glucose was added to distilled water and then dried yeast was added to obtain the mixture solution of glucose and yeast. After five minutes, the powdered Naalginate was mixed with activated yeast. In the second step, the above mixture solution was poured into $1.0 \mathrm{~mm}$ thick glass mold. Subsequently the mold was put into $\mathrm{CaCl}_{2}$ solution at $35{ }^{\circ} \mathrm{C}$, following by cross-linking action of $\mathrm{Ca}^{2+}$. Taking advantage of fermentation action of yeast cells, the instant dry yeast can be used as porogen during the ionic crosslinking process of $\mathrm{SA} / \mathrm{CaCl}_{2}$. Eventually, the obtained hydrogel membrane is a combination of porous structure caused by yeast fermentation and hydrophilic $\mathrm{SA} / \mathrm{CaCl}_{2}$ hydrogel. This complex structure is beneficial for potential application of chemical adsorption and separation. The formation mechanism of porous MIHM samples involves cross-linking of SA/ $\mathrm{CaCl}_{2}$ and fermentation process of yeast/glucose, as shown in Fig. S2. $\dagger$ 


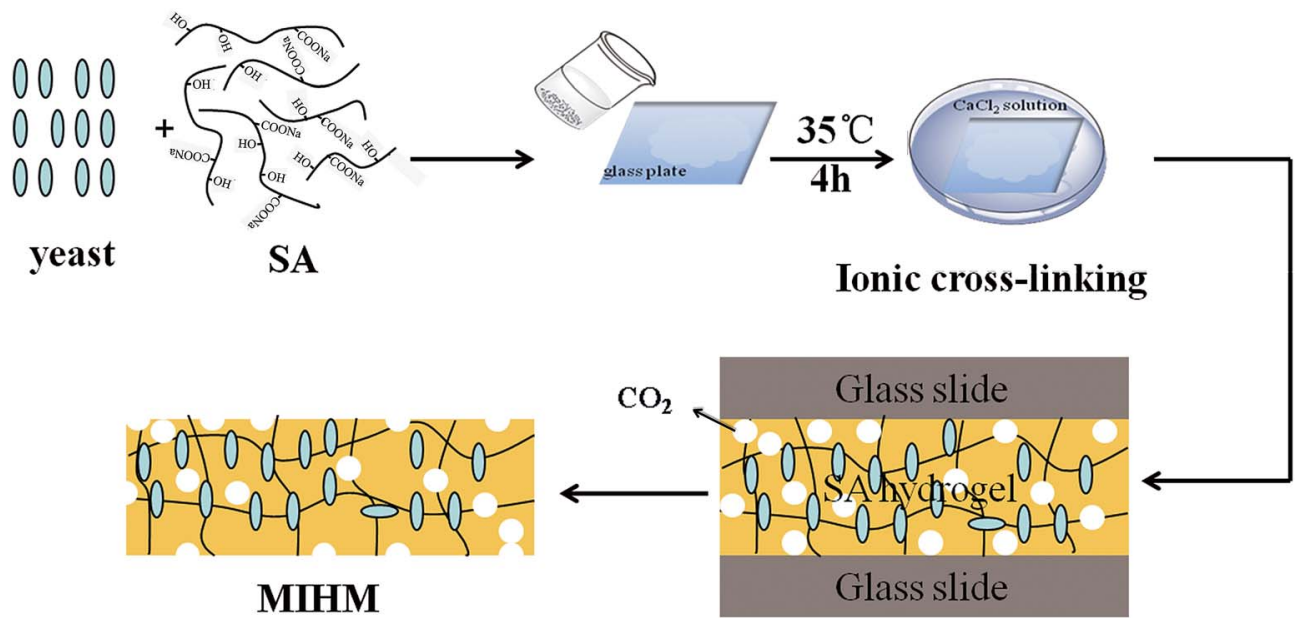

Fig. 2 Schematic representation for the preparation of MIHM hydrogel membranes. The obtained hydrogel membrane is a combination of porous structure caused by yeast fermentation and ionic cross-linked $\mathrm{SA} / \mathrm{CaCl}_{2}$ hydrogel.

\subsection{The pore shape, porosity and pore size of MIHM hydrogel membranes}

To observe the morphology of fabricated MIHM hydrogel membranes, ESEM observation was performed, as shown in Fig. 3. It is seen that pure $\mathrm{SA} / \mathrm{CaCl}_{2}$ hydrogel membrane presents smooth surface and no pore structure could be found on the surface under any magnification. However, for MIHM5 and MIHM15, interconnected micro and macro pores can be observed, indicating that the introduction of yeast/glucose accelerates the formation of porous structure. In terms of macro pores, MIHM5 shows average $0.5 \mathrm{~mm}$ diameter pores, while MIHM15 exhibits larger pores with average $1 \mathrm{~mm}$ diameter and rougher pore wall surface. Actually, with the mass ratios of yeast/SA increased, more $\mathrm{CO}_{2}$ is generated, and hydrogel membranes display larger pore size in light of bubble coalescence. On the other hand, due to increased content of
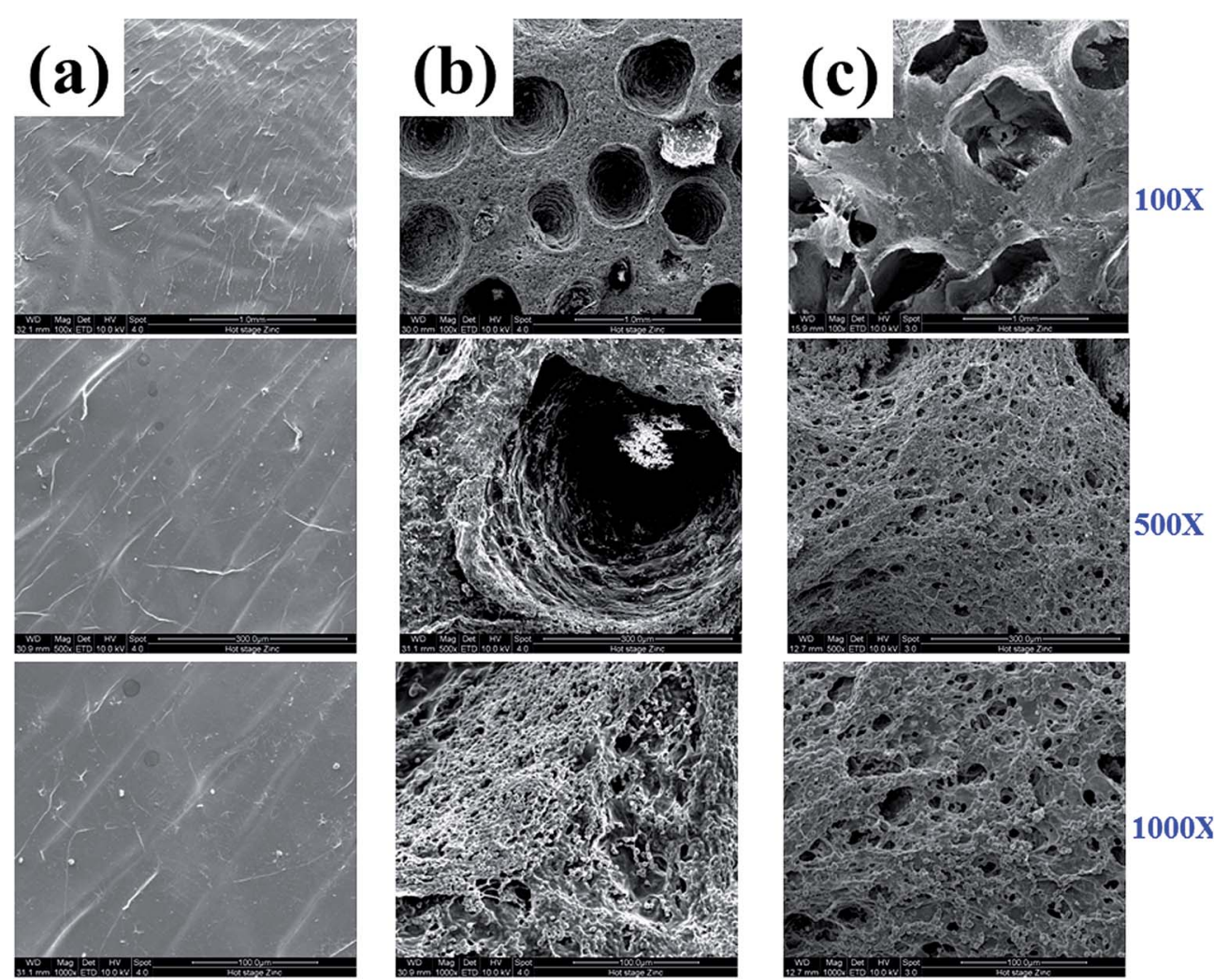

Fig. 3 ESEM images of MIHM hydrogel membranes prepared by cross-linking method from aqueous solutions of $\mathrm{SA}$ and $\mathrm{CaCl}_{2}$ with following ratios of yeast/SA. (a) MIHMO, (b) MIHM5 and (c) MIHM15. 
yeast, the pore wall of MIHM15 becomes denser than that of MIHM5 because large amounts of dead yeast cells deposit inside of the membrane, which can be clearly seen under high magnification of ESEM images shown in Fig. S1. $\dagger$

Fig. 4 shows the FESEM images of cross section of MIHM hydrogel membranes. The membrane specimens were fractured after freeze-dried and coated with a gold layer before observation. It is seen that the cross sections of all MIHM hydrogel membranes present porous structure with diameter ranging from 18 to $200 \mu \mathrm{m}$. Especially for MIHM0 hydrogel membrane, there are a lot of large pores $(100-200 \mu \mathrm{m})$ with a thick pore wall, which is completely different from smooth and compact membrane surface. Compared to MIHM0, the pore diameters of MIHM5 and MIHM15 dramatically decrease to 16-27 $\mu \mathrm{m}$ and the pore size is uniform. The pore structure of cross-section is different from that on the surface which has hierarchical porous structure. Fig. $4 \mathrm{~b}$ and $\mathrm{c}$ also show that massive dead yeast cells adhere to the pore walls and occupy the pore space. Besides, the pore size of MIHM15 is larger than that of MIHM5, which is considered to be due to that higher content of yeast and glucose could produce a larger amount of $\mathrm{CO}_{2}$ during fermentation.

It's known that mercury intrusion porosimetry (MIP) can provide a wide range of information, such as porosity and the specific surface area. Here, the porosity features of MIHM0, MIHM5 and MIHM15 hydrogel membrane were displayed in Table 1. It is seen that, with the mass ratios of yeast/SA increased from 0 to 15 , the porosity of MIHM hydrogel membrane decreases from 81.49 to $44.00 \%$ and pore volume decreases from $6.91 \mathrm{~cm}^{3} \mathrm{~g}^{-1}$ to $2.19 \mathrm{~cm}^{3} \mathrm{~g}^{-1}$. Besides, the pore size of MIHM hydrogel membranes presents a decreasing
Table 1 MIP data of MIHM hydrogel membranes with different mass ratios of yeast/SA

\begin{tabular}{lllll}
\hline & $\begin{array}{l}\text { Porosity } \\
(\%)\end{array}$ & $\begin{array}{l}\text { Total pore area } \\
\left(\mathrm{m}^{2} \mathrm{~g}^{-1}\right)\end{array}$ & $\begin{array}{l}\text { Pore volume } \\
\left(\mathrm{cm}^{3} \mathrm{~g}^{-1}\right)\end{array}$ & $\begin{array}{l}\text { Average pore } \\
\text { diameters }(\mathrm{nm})\end{array}$ \\
\hline MIHM0 & 81.49 & 14.73 & 6.91 & 1874.7 \\
MIHM5 & 66.64 & 19.23 & 5.08 & 1056.4 \\
MIHM15 & 44.00 & 31.23 & 2.19 & 281
\end{tabular}

tendency. For example, MIHM0 exhibits relatively big pores with an average diameter of $1874.7 \mathrm{~nm}$, while MIHM15 has much smaller pores with an average diameter of $281 \mathrm{~nm}$. Compared to pure $\mathrm{SA} / \mathrm{CaCl}_{2}$ hydrogel membrane, the decrease of porosity and pore size caused by the introduction of yeast can be ascribed to the existence of massive dead yeast cells.

It is a fact that MIP can just measure $3.6 \mathrm{~nm}$ to $440 \mu \mathrm{m}$ level pores and its accuracy in measuring small pores is limited due to compressibility of the polymeric matrix itself. SEM graphs have confirmed that MIHM5 and MIHM15 hydrogel membranes possess millimeter scale pores as well as micro pores below $60 \mathrm{~nm}$. To further analyze the pore properties (pore size distribution and cumulative intruded pore volume) and surface area, the freeze-dried samples were measured by BET, as shown in Table 2. Compared to pure $\mathrm{SA} / \mathrm{CaCl}_{2}$ hydrogel membrane (MIHM0) and MIHM5, the surface area and pore diameter of MIHM15 exhibit the highest value, that is $88.77 \mathrm{~m}^{2} \mathrm{~g}^{-1}$ and $17.12 \mathrm{~nm}$, respectively. This is most likely due to the fact that higher content of yeast and glucose could produce more $\mathrm{CO}_{2}$ during fermentation.
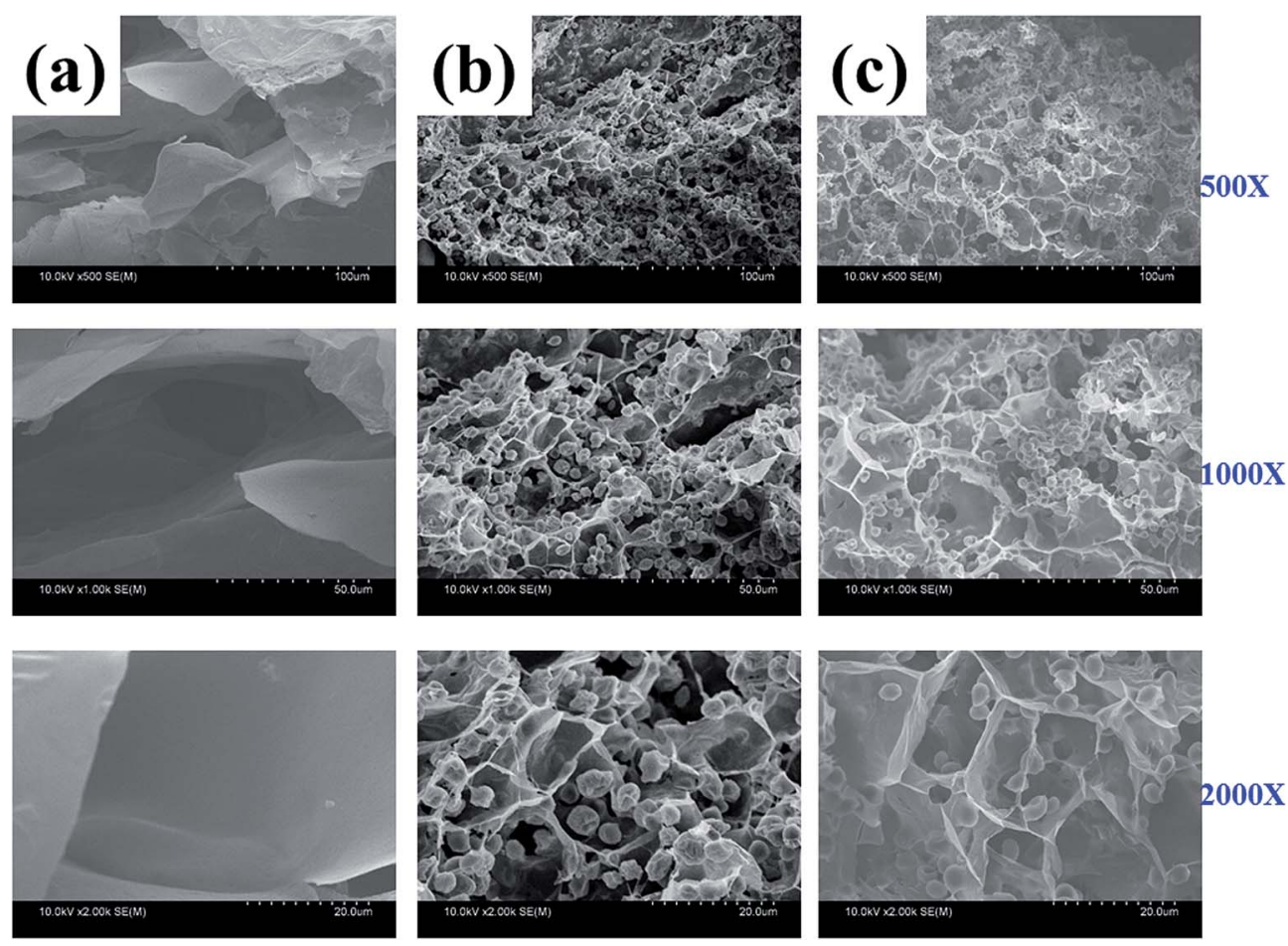

Fig. 4 FESEM images of cross section of MIHM hydrogel membranes under different magnifications. (a) MIHM0, (b) MIHM5, (c) MIHM15. 
Table 2 BET data of MIHM hydrogel membranes with different mass ratios of yeast/SA

\begin{tabular}{llll}
\hline No. & $\begin{array}{l}\text { Total pore volume } \\
\left(10^{-2} \mathrm{~mL} \mathrm{~g}^{-1}\right)\end{array}$ & $\begin{array}{l}\text { Surface area } \\
\left(\mathrm{m}^{2} \mathrm{~g}^{-1}\right)\end{array}$ & $\begin{array}{l}\text { Pore diameter } \\
(\mathrm{nm})\end{array}$ \\
\hline MIHM0 & 2.275 & 12.478 & 4.614 \\
MIHM5 & 7.455 & 40.911 & 1.754 \\
MIHM15 & 9.094 & 88.772 & 17.117
\end{tabular}

From the results of SEM, MIP and BET, it can be assumed that the increased mass ratios of yeast/SA lead to superporous and microporous structure. The reasons can be illustrated by following two competitive aspects. On the one hand, the formation of superporous pores lies in that high content of yeast and glucose could produce a large amount of $\mathrm{CO}_{2}$ during fermentation. On the other hand, the micropores decrease because the introduced yeast exists in the MIHM hydrogel membranes.

\subsection{The surface hydrophilic/hydrophobic property and maximum swelling behavior of MIHM hydrogel membranes}

To investigate hydrophilicity/hydrophobicity of MIHM samples, contact angle (CA) was performed. Fig. 5a shows the CA data and images of MIHM. The CA values of MIHM0, MIHM5 and MIHM15 hydrogel membranes are $63.65^{\circ} \pm 1.45,82.67^{\circ} \pm 2.62$, $107.83^{\circ} \pm 2.79$, respectively. It suggests that the formation of porous structure caused by yeast fermentation increases the hydrophobicity of MIHM hydrogel membranes. In other words, the increases of mass ratios of yeast/SA enhance the hydrophobicity of MIHM hydrogel membranes. As a matter of fact, the hydrophilicity/hydrophobicity of materials depends on the chemical composition and surface structure. Raghu et al. pointed out that the increase of polar groups resulted in an increase of available polar (hydrophilic) groups, which contributed to the formation of more hydrophilic and rougher surface. ${ }^{19}$ In terms of chemical composition of MIHM0, MIHM5 and MIHM15 hydrogel membrane, the concentration of hydrophilic SA and $\mathrm{CaCl}_{2}$ is the same and the main difference lies in the mass ratios of yeast/SA. However, as shown in Fig. 3 and 4, high mass ratios of yeast/SA lead to hierarchical pore structure and dense pore wall, which is the key factor for increasing hydrophobicity of MIHM hydrogel membranes. Pan et al. fabricated hydrophobic polymer foam with hierarchical porous structure $(0.1-0.2 \mu \mathrm{m})$ and found that the contact angle range from $122^{\circ}$ to $136^{\circ} .^{20}$

To further compare the hydrophilicity/hydrophobicity of MIHM hydrogel membranes, maximum swelling ratios (MSR) were obtained, as shown in Fig. 5b. It is seen that the MSR values present a declining curve with the increase of mass ratios of yeast/SA. The pure MIHM0 hydrogel membrane exhibits the highest MSR value, $79.72 \pm 5.43 \mathrm{~g} \mathrm{~g}^{-1}$, while just $23.67 \pm 0.93 \mathrm{~g}$ $\mathrm{g}^{-1}$ and $18.05 \pm 0.52 \mathrm{~g} \mathrm{~g}^{-1}$ for MIHM5 or MIHM15 hydrogel membranes, which are much higher than porous bulk PAM hydrogel. ${ }^{14}$ It indicates that the increased mass ratios of yeast/ SA indeed decrease the MSR value of pure MIHM0 (SA/CaCl 2$)$ hydrogel membrane. In general, the swelling behavior of hydrogel network depends on the osmotic pressure between polymer chains and water molecules and is restricted by crosslinking degree of network flexibility. Hassan et al. pointed out that the free volumes between the macromolecular chains reduced with increasing cross-linking degree in the hydrogel, meanwhile, the diffusion of water into the matrix becomes difficult. ${ }^{21}$ Actually, as shown in Fig. $\mathrm{S} 1, \uparrow$ the added instant yeast cells occupy the network structure space as a kind of filler, preventing water molecules from diffusing into the matrix. Therefore, although MIHM15 hydrogel membrane exhibits hierarchical pore structure, the water capacity and maximum swelling ratio as a whole keep at a low level.

\subsection{The adsorption behavior of MIHM hydrogel membranes onto CV}

Cationic dye $\mathrm{CV}$ belongs to triphenylmethane group and is harmful for the skin, mucous membranes and respiratory system by simple contact, ingestion and inhalation. What's the effect of large pore structure or interconnected capillary channels upon adsorption of cationic dye CV? The membranes with large and numerous pores or interconnected capillary channels may be favorable for CV adsorption. To confirm this, the adsorbing
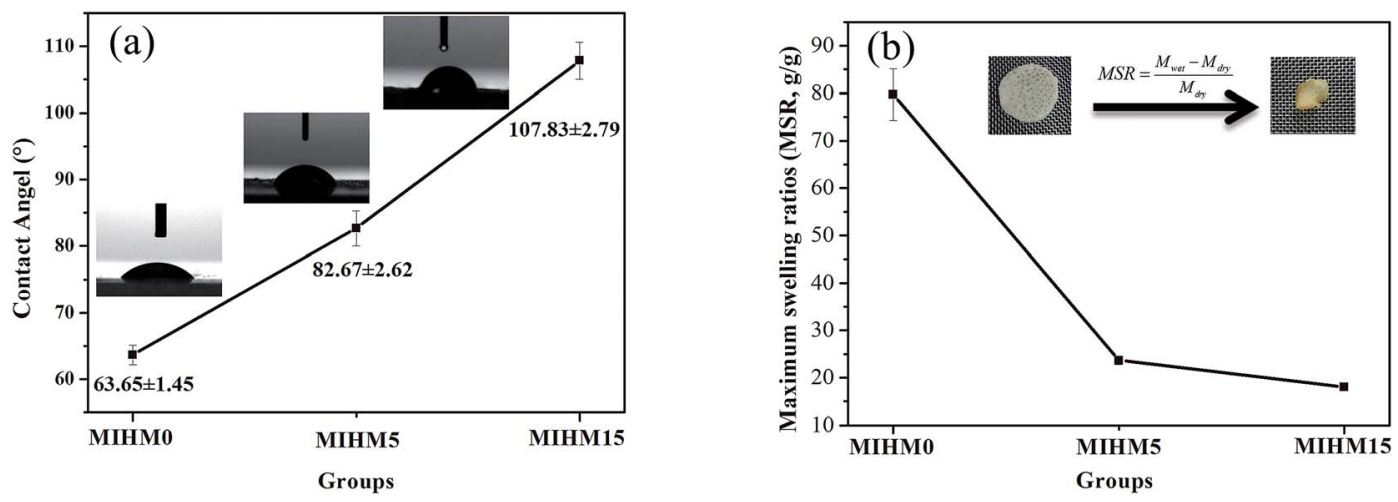

Fig. 5 (a) Contact angle $\left(^{\circ}\right)$ changes and images of MIHM hydrogel membranes with different ratios of yeast/SA showing an increase in hydrophobicity with ratios of yeast/SA increase. Each sample was repeated for three times, (b) maximum swelling ratios (MSR) of MIHM hydrogel membranes with different ratios of yeast/SA from MIHMO to MIHM15 at $25^{\circ} \mathrm{C}$. 
(a)

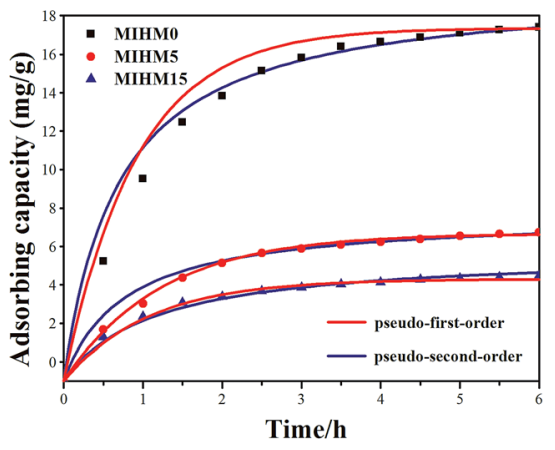

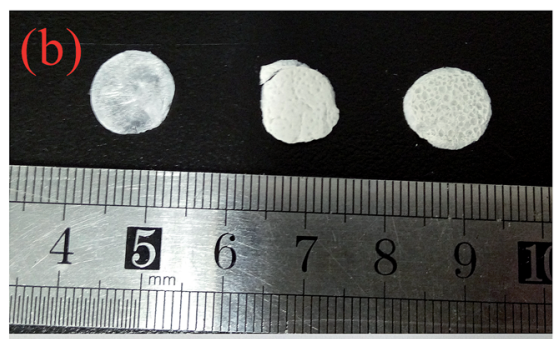

(c)
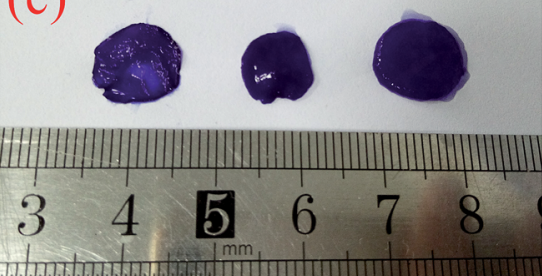

Fig. 6 (a) Effect of contact time on $\mathrm{CV}$ adsorption capacity $\left(10 \mathrm{mg} \mathrm{L}^{-1}\right)$ of prepared MIHM hydrogel membranes. The contact time ranges from 0 to $360 \mathrm{~min}$. MIHMO hydrogel displays the highest adsorption capacity. And curves of pseudo-first-order and pseudo-second-order compared with experimental data, (b) appearance of MIHM hydrogel membranes before adsorption, (c) appearance of MIHM hydrogel membranes after adsorption.

capacities of the porous MIHM hydrogel membranes at different adsorption time at $25^{\circ} \mathrm{C}$ were investigated. Fig. 6 a shows a plot of dye adsorbing capacity $\left(\mathrm{mg} \mathrm{g}^{-1}\right)$ versus contact time for each MIHM at $10 \mathrm{mg} \mathrm{L}{ }^{-1}$ of $\mathrm{CV}$. According to previous reports, the maximum adsorption wavelength of $\mathrm{CV}$ is $584 \mathrm{~nm}$, namely, $\lambda_{\max }=584 \mathrm{~nm}$. The linear relationship between absorbance values $(A)$ of $\mathrm{CV}$ solution and $\mathrm{CV}$ concentration $(C)$ was shown in eqn (3). Combined with eqn (2), the $Q_{t}$ values can be calculated.

$$
A=0.16995 C-0.0483\left(R^{2}=0.99953\right)
$$

It can be found that all MIHM hydrogel membranes present good dependence on adsorption time. With adsorption time extending from 0 to $360 \mathrm{~min}$, the $Q_{t}$ values increase from 0 to $5^{-}$ $9 \mathrm{mg} \mathrm{g}^{-1}$. It is of interest to note that, after $360 \mathrm{~min}$, the $Q_{t}$ value of MIHM5 hydrogel membrane reaches $18.40 \mathrm{mg} \mathrm{g}^{-1}$, which is nearly 2.37 times of MIHM0 and 3.08 times of MIHM15. In other words, as the mass ratio of yeast/SA is 0 , the adsorption amount $\left(Q_{t}\right)$ presents the highest value. The maximum adsorption capacity of MIHM hydrogels is nearly 4 times as high as the PNIPAm/Clay nanocomposite hydrogels and 3-26 times of porous bulk PAM hydrogel previously prepared by our group. ${ }^{16,22}$ The reason can be ascribed to the pore structure and pore shape of MIHM hydrogel membrane as mentioned in 3.3.
On the other hand, as shown in Fig. 6b and c, all MIHM hydrogel samples turn into violet from original white after adsorption. The appearance changes indicate that MIHM hydrogels show good adsorption performance.

MIHM0 displays the highest adsorption capacity, which can be concluded from the facts that it exhibited the lowest weight in the same condition, the largest pore size of cross section seen from Fig. 6 and the largest porosity, pore volume and average pore diameter seen from Tables 1 and 2.

To investigate adsorption kinetics of MIHM samples on CV, two kinds of kinetic models like pseudo-first-order and pseudosecond-order are employed. The best-fit model is selected on the basis of correlation coefficient $R^{2}$ value.

The pseudo-first-order model. According to Lagergren pseudo-first-order kinetics, ${ }^{23}$ the following eqn (4)-(6) can be obtained,

$$
\begin{gathered}
\frac{\mathrm{d} Q_{t}}{\mathrm{~d} t}=k_{1}\left(Q_{\mathrm{e}}-Q_{t}\right) \\
\frac{\mathrm{d}\left(Q_{\mathrm{e}}-Q_{t}\right)}{Q_{\mathrm{e}}-Q_{t}}=-k_{1} \mathrm{~d} t \\
\log \left(\frac{Q_{\mathrm{e}}}{Q_{\mathrm{e}}-Q_{t}}\right)=\frac{k_{1}}{2.303} t
\end{gathered}
$$

\begin{tabular}{|c|c|c|c|c|c|c|c|}
\hline & $Q_{\mathrm{e}}\left(\mathrm{mg} \mathrm{g}^{-1}\right)$ & \multicolumn{3}{|c|}{ Pseudo-first-order kinetic model } & \multicolumn{3}{|c|}{ Pseudo-second-order kinetic model } \\
\hline MIHM5 & 7.75 & 7.68 & 0.0014 & 0.9822 & 5.91 & 0.2908 & 0.9888 \\
\hline MIHM15 & 5.97 & 5.31 & 0.0156 & 0.9783 & 20.53 & 0.0706 & 0.9817 \\
\hline
\end{tabular}

Table 3 Calculated and experimental values $\left(Q_{e}\right)$ for MIHM hydrogel membranes absorbing CV under pseudo-first-order and pseudo-secondorder kinetic models 
where $Q_{t}$ and $Q_{\mathrm{e}}$ represent dye adsorbed quantity $\left(\mathrm{mg} \mathrm{g}^{-1}\right)$ at $t$ time and at equilibrium time. $k_{1}$ represents the adsorption rate constant $\left(\mathrm{min}^{-1}\right)$, which can be calculated from the slope value of the plot of eqn (6). The plot of $\log \left(\frac{Q_{\mathrm{e}}}{Q_{\mathrm{e}}-Q_{t}}\right)$ versus $t$ gives a straight line for the pseudo-first-order kinetics from which the equilibrium sorption capacity $\left(Q_{\mathrm{e}}\right)$ and rate constant can be estimated. According to the fitting result, the adsorption rate constant $k_{1}\left(\mathrm{~min}^{-1}\right)$ and correlation coefficient $R^{2}$ values are shown in Table 3 . The $R^{2}$ values for the plots of pseudo-firstorder are $0.9951,0.9822$ and 0.9783 , respectively, suggesting a proper fitting of the kinetic data into the pseudo-first-order rate law. The adsorbing capacities evaluated from the intercepts are $18.38,7.68$ and $5.31 \mathrm{mg} \mathrm{g}^{-1}$, respectively, for initial concentration of $\mathrm{CV}$ as $10 \mathrm{mg} \mathrm{L}^{-1}$.

The pseudo-second-order model. According to Ho pseudosecond-order kinetics, ${ }^{24}$ the eqn (7)-(9) can be obtained,

$$
\begin{gathered}
\frac{\mathrm{d} Q_{t}}{\mathrm{~d} t}=k_{2}\left(Q_{\mathrm{e}}-Q_{t}\right)^{2} \\
\frac{t}{Q_{t}}=\frac{1}{k_{2} Q_{\mathrm{e}}{ }^{2}}+\frac{t}{Q_{\mathrm{e}}} \\
h=k_{2} Q_{\mathrm{e}}{ }^{2}(t \rightarrow 0)
\end{gathered}
$$

where $Q_{t}$ and $Q_{\mathrm{e}}$ represent dye adsorbed quantity $\left(\mathrm{mg} \mathrm{g}^{-1}\right)$ at $t$ time and at equilibrium time, and $k_{2}$ represents the adsorption rate constant $\left(\mathrm{g} \mathrm{mg}^{-1} \mathrm{~h}^{-1}\right)$.

As shown in Table 3, the correlation coefficients for pseudosecond-order of different MIHM porous hydrogel membranes are $0.9789,0.9888$ and 0.9817 . It reveals that both the pseudofirst-order model and pseudo-second-order fit for the MIHM adsorption on CV since the correlation coefficients $\left(R^{2}\right)$ are close to 1 . However, the calculated values of $Q_{\mathrm{e}}$ of pseudo-first-order model are quite close to the experimental values. Therefore, the adsorption should belong to physical adsorption. Besides, the adsorbent and $\mathrm{CV}$ have nearly no groups to react. The similar phenomena are also observed in adsorption of dye RB4 and toxic metals on modified chitosan. ${ }^{25}$

\section{Conclusions}

In this work, by combining ionic cross-linking method with fermentation technology, the super/macro-porous calcium alginate hydrogel membranes with hierarchical pore structure were prepared under different mass ratios of yeast/SA. The obtained Microorganism Inspired Hydrogel Membranes (MIHM), via this simple and versatile method for generating large amounts of bubbles, presented hierarchical porous structure ranging from $2 \mathrm{~nm}$ to $1 \mathrm{~mm}$ and increasing absorbance value from 1.95 to 2.63. More importantly, the formation of porous structure caused by yeast fermentation increased the hydrophobicity of MIHM and decreased the maximum swelling ratios. As a remover of $\mathrm{CV}$ dye, the maximum adsorption capacity of the MIHM hydrogel membrane is $8.78 \mathrm{mg} \mathrm{g}^{-1}$ with the initial concentration of $10 \mathrm{mg} \mathrm{L}^{-1}$. Depending on the weight and porosity, the adsorption capacity of MIHM hydrogel membranes at $10 \mathrm{mg} \mathrm{L}^{-1}$ of $\mathrm{CV}$ ranged from 5.97 to $18.40 \mathrm{mg}$ $\mathrm{g}^{-1}$, illustrating the optimal pore size of MIHM was related to that of CV. Kinetic studies showed that adsorption of dispersed dyes could be well defined by pseudo-first-order kinetic equation with a high correlation coefficient $\left(R^{2}>0.97\right)$. This work established a solid foundation for future work to obtain porous hydrogel membranes by fermentation method.

\section{Conflicts of interest}

The authors declared that they have no conflicts of interest to this work. We declare that we do not have any commercial or associative interest that represents a conflict of interest in connection with the work submitted.

\section{Acknowledgements}

This work has been supported by Applied Basic Research and Advanced Technology Programs of Science and Technology Commission Foundation of Tianjin (12JCQNJC01400 \& 15JCYBJC18300), Science and Technology Correspondent of Tianjin (14JCTPJC00502 \& 15JCPJC62200).

\section{References}

1 L. Wang and Y. Ding, Eur. Polym. J., 2015, 66, 99-107.

2 M. De Colli, M. Massimi, A. Barbetta, B. L. Di Rosario, S. Nardecchia, L. Conti Devirgiliis and M. Dentini, Biomed. Mater., 2012, 7, 1465-1476.

3 I. Tokarev, V. Gopishetty and S. Minko, ACS Appl. Mater. Interfaces, 2015, 7, 12463-12469.

4 H. Bai, A. Polini, B. Delattre and A. P. Tomsia, Chem. Mater., 2013, 25, 4551-4556.

5 W. Wang, L. Zong and A. Wang, Int. J. Biol. Macromol., 2013, 62, 225-231.

6 B. Gyarmati, E. Z. Meszar, L. Kiss, M. A. Deli, K. Laszlo and A. Szilagyi, Acta Biomater., 2015, 22, 32-38.

7 T. Agarwal, S. N. Narayana, K. Pal, K. Pramanik, S. Giri and I. Banerjee, Int. J. Biol. Macromol., 2015, 75, 409-417.

8 E. S. Chan, S. L. Wong, P. P. Lee, J. S. Lee, T. B. Ti, Z. Zhang, D. Poncelet, P. Ravindra, S. H. Phan and Z. H. Yim, Carbohydr. Polym., 2011, 83, 225-232.

9 J. H. Lin, C. K. Chen, S. P. Wen and C. W. Lou, Mater. Sci. Eng., C, 2015, 52, 111-120.

10 P. S. Rao, A. Krishnaiah, B. Smitha and S. Sridhar, Sep. Sci. Technol., 2006, 41, 979-999.

11 J. Xu, J. Membr. Sci., 2004, 240, 81-89.

12 R. V. Kulkarni, V. Sreedhar, S. Mutalik, C. M. Setty and B. Sa, Int. J. Biol. Macromol., 2010, 47, 520-527.

13 X. X. Zhang, B. B. Lin, K. Y. Zhao, J. F. Wei, J. Guo, W. K. Cui, S. Jiang, D. Liu and J. X. Li, Desalination, 2015, 365, 234-241. 14 Q. S. Zhang, B. J. Chen, L. Tao, M. Y. Yan, L. Chen and Y. Wei, RSC Adv., 2014, 4, 32475-32481.

15 B. J. Chen, S. H. Zhang, Q. S. Zhang, Q. F. Mu, L. L. Deng, L. Chen, Y. Wei, L. Tao, X. Y. Zhang and K. Wang, RSC Adv., 2015, 5, 91937-91945. 
16 G. A. Junter and F. Vinet, Chem. Eng. J., 2009, 145, 514-521. 17 G. A. Junter, D. T. Bui and F. Vinet, Chem. Eng. J., 2009, 152, 297-300.

18 W. J. Gao, Q. S. Zhang, P. F. Liu, S. H. Zhang, J. Zhang and L. Chen, RSC Adv., 2014, 4, 34460-34469.

19 D. P. Suhas, T. M. Aminabhavi and A. V. Raghu, Appl. Clay Sci., 2014, 101, 419-429.

20 J. Pan, Y. Mao, H. Gao, Q. Xiong, F. Qiu, T. Zhang and X. Niu, Carbohydr. Polym., 2016, 143, 212-222.
21 G. A. Mahmoud, S. F. Mohamed and H. M. Hassan, Desalin. Water Treat., 2014, 54, 2765-2776.

22 Q. S. Zhang, T. T. Zhang, T. He and L. Chen, Appl. Clay Sci., 2014, 90, 1-5.

23 V. Vadivelan and K. V. Kumar, J. Colloid Interface Sci., 2005, 286, 90-100.

24 Y. S. Ho and G. Mckay, Process Biochem., 1999, 34, 451-465. 25 V. Dhanapal and K. Subramanian, Carbohydr. Polym., 2015, 117, 123-132. 